\title{
PROCEEDINGS OF THE DUTCH SOCIETY OF CLINICAL PHARMACOLOGY AND BIOPHARMACY MEETING OF SEPTEMBER 27TH 2006
}

\section{These abstracts have been accepted for publication by the Dutch Society of Clinical Pharmacology and Biopharmacy}

\section{Acute MDMA and ethanol interaction effects on psychomotor performance \\ G. J. H. Dumont, ${ }^{1}$ M. M. G. J. Valkenberg, ${ }_{1}^{1}$ R. Schoemaker, ${ }^{2}$ \\ J. K. Buitelaar, ${ }^{1}$ J. M. A. van Gerven ${ }^{2}$ \& R. J. Verkes' \\ 'Unit for Clinical Psychopharmacology and Neuropsychiatry (UCPN), Dept. Psychiatry, University Medical Centre Nijmegen, \\ The Netherlands ${ }^{2}$ Centre for Human Drug Research, Leiden, \\ The Netherlands}

Introduction: A considerable percentage of young people expose themselves to 3,4-methylenedioxy-methamphetamine (MDMA or 'ecstasy'). Alcohol is relatively commonly used in combination with MDMA (Barrett et al., 2005). As a percentage of MDMA users admit to driving under influence (Riley et al., 2001), the assessment of the effects of co-administration of these substances on psychomotor performance is warranted.

Hypothesis: We hypothesized that the co-administration of MDMA and ethanol would ameliorate the impairment induced by ethanol alone.

Methods: We performed a double-blind, randomized, placebocontrolled study in 16 healthy volunteers ( 9 male, 7 female) in the age of 18-29 years. MDMA $100 \mathrm{mg}$ was given orally and blood alcohol concentration (BAC) was maintained at $0.6 \%$ by a three hour $10 \%$ alcohol infusion regime (adjustment of infusion rate according to regular sampled breath alcohol concentration). Saccadic and smooth pursuit eye movements, a validated measure of ethanol induced sedation (van Steveninck et al., 1999), were recorded using
Nihon Kohden ${ }^{\circledR}$ and Cambridge Electronic Design $(C E D \circledR)$ hardware and Spike2 ${ }^{\circledR}$ software at $1: 35$ post drug administration. Results were statistically evaluated using GLM in SPSS ${ }^{\circledR}$ 11.5.

Results: Results are presented in table 1. Saccadic peak velocity was increased after MDMA ( $\mathrm{p}=0.000)$. Co-administration showed significant increase in peak saccadic velocity compared to placebo ( $\mathrm{p}=$ 0.004), although significantly less then the increase as observed after MDMA alone $(p=0.003)$. Smooth pursuit was significantly impaired after ethanol compared to placebo $(\mathrm{p}=0.000)$ and MDMA $(\mathrm{p}=$ $0.000)$, co-adminstration also impaired score compared to placebo $(\mathrm{p}=0.000)$ and MDMA $(\mathrm{p}=0.000)$.

Conclusions: MDMA induced arousal as measured by the saccadic peak velocity when administered alone and, to a lesser degree, in combination with ethanol. Co-administration of MDMA with ethanol however could not ameliorate the deterioration of psychomotor accuracy by ethanol as measured by smooth pursuit eye movements. These findings suggest that although MDMA might ameliorate ethanol induced sedation it does not ameliorate impaired psychomotor skills. This might give a false sense of improved performance while intoxicated and thus increases risks of for example road accidents.

Barrett et al. Subst. Use. Misuse 2005; 40: 1525-1537.

Riley et al. Addiction 2001; 96: 1035-1047.

Van Steveninck et al. J. Psychopharmacol 1999; 13: 10-17.
Table 1

Eye movement scores per drug

\begin{tabular}{|c|c|c|c|c|c|c|c|c|}
\hline \multirow[b]{2}{*}{ Eye movements } & \multicolumn{2}{|c|}{ Placebo } & \multicolumn{2}{|c|}{ Ethanol } & \multicolumn{2}{|c|}{ MDMA } & \multicolumn{2}{|c|}{$\begin{array}{l}\text { Ethanol + } \\
\text { MDMA }\end{array}$} \\
\hline & Mean & SD & Mean & SD & Mean & SD & Mean & SD \\
\hline Peak saccadic & & & $\bullet$ & & $*, \bullet$, & & $*, \bullet, \bullet$ & \\
\hline velocity (deg/s) & 458,1 & 66,1 & 452,8 & 54,8 & 543,5 & 76,3 & 497,2 & 65,8 \\
\hline $\begin{array}{l}\text { Saccadic reaction } \\
\text { time }(\mathrm{ms})\end{array}$ & 190,8 & 25,3 & 194,4 & 26,1 & 193,0 & 24,9 & 191,6 & 24,9 \\
\hline Average smooth & & & $*_{1}$ & & $\bullet$ & & $*_{,}$ & \\
\hline pursuit (\%) & 49,08 & 12,00 & 37,56 & 9,72 & 52,74 & 14,30 & 38,90 & 9,85 \\
\hline
\end{tabular}

* = Significant vs. placebo, $\bullet=$ Significant vs. ethanol, $\bullet=$ Significant vs. MDMA, $\bullet$ Significant vs. MDMA + ethanol. 


\section{A polypharmacy intervention study on Dutch nursing home residents}

\author{
F. Finkers, ${ }^{1}$ J. G. Maring, ${ }^{2}$ F. Boersma ${ }^{3}$ \& K. Taxis ${ }^{1}$ \\ 'Department of Social Pharmacy, Pharmacoepidemiology and \\ Pharmacotherapy, Groningen University Institute for Drug \\ Exploration Groningen. ${ }^{2}$ Department of Pharmacy, Diaconessen \\ Hospital Meppel \& Bethesda Hospital, Meppel. ${ }^{3}$ Department of \\ Internal Medicine and Geriatrics, University Medical Centre \\ Groningen, University of Groningen, The Netherlands
}

Objectives: This study firstly aimed to identify the percentage of nursing home patients who were prescribed more than nine drugs (polypharmacy) and compared prescribing patterns of this patient group to patients who were prescribed fewer drugs. Secondly, teams of hospital pharmacists and nursing home physicians aimed to identify the drug related problems of the polypharmacy patients and the extent to which it was feasible to optimise their medication profiles.

Methods: Characteristics of the polypharmacy population were illustrated by retrospective point measurements in five Dutch nursing homes (total of 742 beds) over the years 2004, 2005 and 2006. Additionally, 91 polypharmacy patients in those nursing homes were included for a medication review by a hospital pharmacist and a nursing home physician. The appropiateness of each drug was discussed adressing indication, effectiveness and safety, as well as possible undertreatment. Drug related problems were accordingly (sub)categorised. The pharmacist and physician prioritised the problems and jointly preparared a plan who to optimise the medication profile.
Six weeks later, the same pharmacist and physician met again and discussed for each patient the list of problems and the alterations in the medication profile.

Results: We found an increase in the percentage polypharmacy patients over the years $(14.6 \%, 2004 ; 17.5 \%, 2005 ; 19.8 \%, 2006)$. Drugs from the ATC-groups 'alimentary tract and metabolism', 'cardiovascular system' and 'nervous system' were prescribed most frequently in the polypharmacy population. The 91 reviewed polypharmacy patients used 1226 drugs. We registered 323 drug related problems (mean of 3.5 problems per patient). A decreased renal function (creatinin clearance $<40 \mathrm{ml} / \mathrm{min}$ ) was observed in a high number of polypharmacy patients (at least $40 \%$ ). The majority of drug related problems were in the category 'indication'. In total, $62 \%$ of all drug related problems were subcategorised as 'unclear or not confirmed indication'. These problems were observed in $87 \%$ of the polypharmacy patients.

A mean of $1.7(n=159)$ problems per patient were solved during the subsequent intervention period and the number of drugs per patients decreased statistically significantly from 13.5 to 12.7 ( $\mathrm{p}<$ 0.0001).

Conclusion: The percentage of nursing home patients meeting the polypharmacy definition ranged from $14,6 \%$ to $19,8 \%$ over 2004-2006. The majority of polypharmacy patients had at least one drug prescribed for which the indication was unknown. The intervention was accompanied with a significant decrease in the number of drugs per patient, but half of the drug related problems remained unsolved.

\section{The potential value on medication safety of a clinical decision support system in intensive care patients with renal insufficiency}

P. J. Helmons, ${ }^{1}$ R. J. E. Grouls, ${ }^{1}$ A. N. Roos, ${ }^{2}$ A. J. G. H. Bindels, ${ }^{2}$

P. A. De Clercq, ${ }^{3}$ S. J. W. Wessels-Basten, ${ }^{1}$ E. W. Ackerman' \&

H. H. M. Korsten ${ }^{4}$

'Departments of Pharmacy, ${ }^{2}$ Internal Medicine and

${ }^{4}$ Anesthesiology, Catharina Hospital Eindhoven, PO Box 1350,

5602 ZA Eindhoven, The Netherlands and ${ }^{3}$ Medecs BV, Horsten

2, 5612 AX Eindhoven, The Netherlands

Introduction: Clinical decision support systems (CDSS) are defined as electronic or non-electronic systems designed to aid in clinical decision making, using characteristics of individual patients to generate patient-specific assessments or recommendations that are then presented to clinicians for consideration (Garg et al., 2005). As the potential value of a CDSS is determined by the benefits on clinical practice of the clinical rules used in this system, it is essential to investigate the necessity and potential benefits on quality of care of these rules (Stiell et al., 1999; Helmons et al., 2006).

Purpose The aim of this study was to determine the potential value of a clinical rule designed to improve dosage adjustment of antibiotics in critically ill patients with renal dysfunction.

Methods: We developed a clinical rule assisting the physicians in selecting the appropriate dosage according to renal function of the ten most frequently prescribed antibiotics. We compared the actual number of dosage adjustments without the support of the CDSS with the theoretical number of dosage adjustments determined by the clinical rule in patients with moderate (creatinin clearance $\left(\mathrm{Cl}_{\text {creat }}\right) 10-50$ $\mathrm{ml} / \mathrm{min})$ and severe $\left(\mathrm{Cl}_{\text {creat }}<10 \mathrm{ml} / \mathrm{min}\right)$ renal dysfunction.

If dosage adjustment was omitted, we determined the overexposure to the antibiotics in terms of duration and number of dosages administered and the extra drug costs involved.

Results: Seventeen hundred eighty-eight patients were included in this retrospective study. In the moderate renal failure group, dosage adjustment was omitted in 163 patients (86\%). In the severe renal failure group, dosage was not adjusted in 13 patients $(54 \%)$. The duration of exposure was the most in patients receiving fluconazole and ciprofloxacin (median duration of 6 days). By adjusting the dosage of the ten most frequently prescribed antibiotics according to renal function, more than $€ 16,000$ can be saved annually on our ICU.

Conclusion: Dosage adjustment of antibiotics is often omitted in our ICU. We conclude that substantial savings and improvement in medication safety can be achieved by implementing this clinical rule in clinical practice. This technology has enormous potential to reduce medication errors. Further (prospective) research is needed to show that this system is clinically applicable.

Garg AX et al. JAMA 2005; 293(10): 1223-38.

Helmons PJ et al. Pharm Weekbl 141(25): 853-7 (Dutch).

Stiell IG et al. Ann Emerg Med 1999; 33(4): 437-47. 


\section{Population PKPD of paclitaxel and carboplatin in ovarian cancer patients: A study by the EORTC-PAMM-NDDG}

M. Joerger, ${ }^{1,2}$ A. D. R. Huitema, D. J. Richel, C. Dittrich,

N. Pavlidis, E. Briasoulis, J. B. Vermorken, E. Strocchi, A. Martoni,

R. Sorio, H. P. Sleeboom, M. A. Izquierdo, D. I. Jodrell,

A. H. Calvert, A. Boddy, H. Hollema. R. Féty, W. J. F. Van der Vijgh,

G. Hempel, E. Chatelut, M. Karlsson, B. Tranchand,

A. H. G. J. Schrijvers, J. H. Beijnen \& J. H. M. Schellens ${ }^{2,3}$

'Department of Pharmacy \& Pharmacology, Slotervaart

Hospital/The Netherlands Cancer Institute, Amsterdam, The

Netherlands, ${ }^{2}$ Department of Medical Oncology, Antoni van

Leeuwenhoek Hospital/The Netherlands Cancer Institute,

${ }^{3}$ Division of Drug Toxicology, Department of Biomedical

Analysis, Faculty of Pharmaceutical Sciences, Utrecht University,

Utrecht, The Netherlands

Introduction: The paclitaxel/carboplatin doublet is standard treatment in advanced ovarian cancer and usually applied following cytoreductive surgery. Paclitaxel displays nonlinear PK, and the exposure-toxicity relationship has been described by thresholdmodels, whereas the time above a paclitaxel plasma concentration of $0.05-0.2 \mu \mathrm{mol} / \mathrm{L}\left(\mathrm{T}_{>\mathrm{C} 0.05}-\mathrm{T}_{>\mathrm{C} 0.2}\right)$ was correlated with hematological toxicity. A semimechanistic PKPD model has recently been described for paclitaxel-induced myelosuppression. The aim of this study was to investigate the population PKPD of paclitaxel and carboplatin in ovarian cancer patients.

Methods: 139 ovarian cancer patients received paclitaxel $175 \mathrm{mg} / \mathrm{m}^{2}$ over three hours, followed by carboplatin AUC $5 \mathrm{mg} * \mathrm{~min} / \mathrm{mL}$ over $30 \mathrm{~min}$, after cytoreductive surgery. Plasma concentration-time data of paclitaxel and carboplatin were measured, and the relationship between PK, hematological toxicity and drug response was evalu- ated using nonlinear mixed-effect modelling. Data were analysed according to a semiphysiological model, including proliferation -, transit - and circulation compartments for neutrophils and thrombocytes. Linear and sigmoidal maximum response models were tested for the influence of paclitaxel and carboplatin on the proliferation rate of neutrophils or thrombocytes. Toxicity and clinical outcome data were analysed by previously established threshold models.

Results: 105 patients had complete PK and toxicity data. In 34 patients with measurable disease, objective response rate was $76 \%$. Hematological toxicity was adequately described by an inhibitory linear drug effect model. Mean paclitaxel $\mathrm{T}_{>00.1}$ was $16.4 \mathrm{hrs}$, mean $\mathrm{T}_{>\mathrm{c} 0.05} 61.4 \mathrm{hrs}$. Patients with a complete response to paclitaxel/carboplatin had a higher $\mathrm{T}_{>\mathrm{C} 0.05}$ as compared to patients with progressive disease ( 91.9 vs $31.5 \mathrm{hrs}, \mathrm{p}=0.02$ ). Similarly, $\mathrm{T}_{>00.05}$ was higher in patients with at least stable as compared to progressive disease (78.3 vs $31.5 \mathrm{hrs}, \mathrm{p}=0.05$ ). Patients with $\mathrm{T}_{>00.05}>61.4 \mathrm{hrs}$ (mean value) had a longer time-to-disease-progression compared to patients with $\mathrm{T}_{>\mathrm{c} 0.05}<61.4$ hours $(89.0$ vs 61.9 weeks, $\mathrm{p}=0.05)$. $\mathrm{T}_{>00.05}$ was also a good predictor for neutropenia, in that patients with absent or mild (WHO grade 1 or 2) neutropenia had a lower $\mathrm{T}_{>00.05}$ as compared to patients with severe (WHO grade 3 or 4 ) neutropenia (50.3 vs $74.1 \mathrm{hrs}, \mathrm{p}=0.01$ ). Carboplatin drug exposure was the best predictor for thrombocytopenia, in that patients with absent thrombopenia had lower values for $\mathrm{C}_{\max }$ and AUC as compared to patients experiencing mild or severe thrombopenia (82.6 vs $132 \mu \mathrm{mol} / \mathrm{L}$ for $\mathrm{C}_{\max }, 1.24$ vs $1.79 \mathrm{mg} * \mathrm{~min} / \mathrm{mL}$ for AUC, $\mathrm{p}<10^{-4}$ for both comparisons).

Conclusions: Paclitaxel $\mathrm{T}_{>\mathrm{c} 0.05}$ was useful to predict neutropenia and clinical outcome in ovarian cancer patients receiving paclitaxel/carboplatin, while carboplatin exposure was useful to predict thrombocytopenia. Prospective studies should assess the value of therapeutic drug monitoring in this patient group.

\section{Dipyridamole enhances ischemia-induced reactive hyperemia by increased adenosine receptor stimulation.}

P. Meijer, ${ }^{1,2}$ N. Riksen, ${ }^{1,3}$ G.-J. Scheffer, ${ }^{2}$ P. Smits ${ }^{1,3}$ \&

G. A. Rongen ${ }^{1,3}$

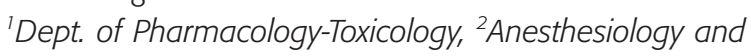

${ }^{3}$ General Internal Medicine, Radboud University Nijmegen

Medical Centre, The Netherlands

Introduction: Dipyridamole enhances post-occlusive reactive hyperemia (PORH) in the human forearm. The mechanism of this potentiation remains to be elucidated. We hypothesize that dipyridamole augments PORH by increasing interstitial adenosine and subsequent stimulation of adenosine receptors. To test this hypothesis, the effect of caffeine (an adenosine A1 and A2 receptor antagonist) on dipyridamole-induced augmentation of PORH was explored.

Methods: Eight healthy volunteers (6 female; age 19-24 year) abstained from caffeine intake for at least 24 hours. The brachial artery was cannulated for drug infusion. After forearm circulation had been occluded for 5 minutes and starting immediately upon reperfusion, forearm blood flow was measured for 5 minutes using venous occlusion mercury-in-silastic strain gauge plethysmography. At least one hour after reperfusion, this procedure was repeated during intra-arterial infusion of dipyridamole $(7.4 \mathrm{nmol} / \mathrm{min} / \mathrm{dl}$ forearm). At least two weeks later, this whole procedure was repeated, but now in the presence of caffeine $(0.46 \mu \mathrm{mol} / \mathrm{min} / \mathrm{dl}$ forearm).

Statistics: For each subsequent minute, forearm blood flow was averaged to one value. An ANOVA for repeated measures was performed with caffeine, dipyridamole and reperfusion time as within-subject factors. Results are expressed as mean \pm SEM.

Results:
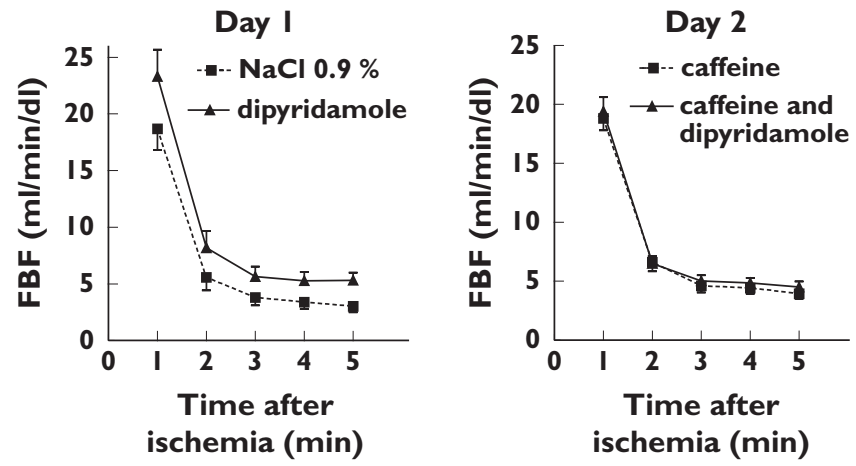
FBF: forearm blood flow

$\mathrm{P}<0.05$ for the effect of dipyridamole on PORH

$\mathrm{P}>0.5$ for the effect of caffeine on PORH

$\mathrm{P}<0.01$ for the interaction between caffeine and dipyridamole.
Conclusions: Caffeine abolishes the augmenting effect of dipyridamole on PORH. This indicates that adenosine receptors are involved in the dipyridamole-induced augmentation of PORH.

\section{Augmented hyperemia and reduced tissue injury in response to ischemia in subjects with the $34 \mathrm{C}>\mathrm{T}$ variant of the AMPD1 gene}

N. P. Riksen, ${ }^{1,2}$ B. Franke, ${ }^{3}$ W. J. G. Oyen, ${ }^{4}$ P. van den Broek, ${ }^{1}$ O. C. Boerman, ${ }^{4}$ P. Smits ${ }^{1,2} \&$ G. A. Rongen ${ }^{1,2}$

'Departments of Pharmacology-Toxicology, ${ }^{2}$ Internal Medicine, ${ }^{3}$ Human Genetics, and ${ }^{4}$ Nuclear Medicine, Radboud University

Nijmegen Medical Center, PO Box 9101, 6500 HB Nijmegen,

The Netherlands.

Introduction: In patients with cardiovascular disease the $34 \mathrm{C}>\mathrm{T}$ variant of the adenosine monophosphate deaminase (AMPD1) gene is associated with improved survival. We hypothesized that this variant results in enhanced intracellular formation of adenosine during ischemia, thereby increasing hyperemia and reducing tissue injury in response to ischemia. As increased intracellular adenosine formation would decrease the transmembranous adenosine concentration gradient, we hypothesized that the potentiating effect of the adenosine transport blocker dipyridamole on reactive hyperemia would be reduced in the subjects with the CT genotype.

Methods: We studied the forearm vasodilator response to ischemia (venous occlusion plethysmography) in 10 healthy subjects with the CT genotype and in $10 \mathrm{CC}$ controls, matched for all relevant parameters of adenosine transport and metabolism. Reactive hyperemia was measured with and without concomitant administration of the adenosine transport blocker dipyridamole into the brachial artery $(7.4 \mathrm{nmol} / \mathrm{min} / \mathrm{dl}$ of forearm tissue).
In comparable groups, ischemia-reperfusion injury was assessed in the thenar muscle by ${ }^{99 \mathrm{~m}} \mathrm{Tc}$-annexin $\mathrm{A} 5$ scintigraphy after intermittent forearm exercise during 10 minutes of ischemia. At reperfusion ${ }^{99 \mathrm{~m}} \mathrm{Tc}$-annexin A5 was administered intravenously. Targeting of annexin was expressed as percentage difference between experimental and contralateral thenar muscle.

Results: Forearm blood flow (FBF) in response to 2 and 5 minutes of ischemia was higher in the CT genotype group than in controls, whereas maximum vasodilation (induced by 13 minutes of ischemia) did not differ $(25.4 \pm 2.5,32.7 \pm 2.2$, and $38.6 \pm 2.6 \mathrm{ml} / \mathrm{min} / \mathrm{dl}$ in the CT group versus $21.9 \pm 2.2,28.5 \pm 2.4$, and $41.0 \pm 3.3 \mathrm{ml} / \mathrm{min} / \mathrm{dl}$ in controls, $\mathrm{P}=0.03$ ).

Dipyridamole potentiated reactive hyperemia in both groups, but this effect of dipyridamole was significantly less in the subjects with the CT genotype $(\mathrm{P}=0.04)$.

At 1 and 4 hours post-injection, annexin targeting was $5.2 \pm 1.8 \%$ and $3.5 \pm 2.3 \%$ in the $\mathrm{CT}$ group versus $8.9 \pm 3.4 \%$ and $9.8 \pm 3.9 \%$ in the controls $(\mathrm{n}=7 ; \mathrm{P}=0.03)$.

Conclusions: The $34 \mathrm{C}>\mathrm{T}$ variant in the AMPD1 gene augments vasodilation and reduces tissue injury in response to forearm skeletal muscle ischemia. Most probably, this is due to increased intracellular adenosine formation, which decreases cellular uptake of adenosine which is formed in the extracellular compartment. These mechanisms could contribute to the survival benefit of cardiovascular patients with this variant allele.

\section{Drug clearance during Continuous Veno-Venous Hemofiltration (CVVH). A mathematical model based on ex-vivo experiments.}

C. M. Samson-Corbeij,, R. J. E. Grouls, ' A. Gerards, ${ }^{1}$

E. W. Ackerman, ${ }^{1}$ P. Douwes-Draaijer, ${ }^{2}$ A. N. Roos ${ }^{2} \&$

A. J. G. H. Bindels ${ }^{2}$

Dept. of 'Clinical Pharmacy and 'Internal Medicine, Catharina

Hospital Eindhoven, PO Box 1350, 5602 ZA Eindhoven, The

Netherlands

Introduction: Continuous veno-venous hemofiltration (CVVH) with an AN69 membrane is used in hemodynamical unstable patients with acute renal failure admitted to the Intensive Care Unit (ICU) of the Catharina Hospital in Eindhoven (CHE), the Netherlands. Literature search revealed that only the clearance and kinetics of some antibiotics during CVVH were studied. Data obtained with different renal replacement techniques and membranes can't easily be extrapolated to CVVH with an AN69 membrane. (Reetze-Bonorden et al. 1993; Mueller et al., 2003) Due to the lack of clinical research data, dosages in patients treated with CVVH at the ICU of the CHE or are not adjusted or are adjusted on the base of the empirical determined creatinin clearance of $30 \mathrm{ml} / \mathrm{min}$. For aminoglycosides and vancomycin dose adjustment is performed applying therapeutic drug monitoring. Because of this a lot of drugs are possibly suboptimal dosed during CVVH.

Aim: The aim of this study was to develop a predictive mathematical model based on ex-vivo experiments for the drug clearance by CVVH with an AN69 high-flux membrane.

Methods: The experimental set-up consisted of a PRISMA CFM hemofiltration machine with a AN69 HF 0,9 m², $500 \mathrm{ml}$ heparinised bovine blood as blood compartment, blood flow rate $150 \mathrm{ml} / \mathrm{min}$ and ultrafiltration rate $1500 \mathrm{ml} / \mathrm{h}$. The following drugs were added to the blood compartment in therapeutic concentrations, covering a broad range in molecular weight $(\mathrm{Mw}), \%$ protein binding $(\% \mathrm{~PB}), \log \mathrm{P}$ and charge $(\mathrm{C})$ : amiodarone, amitriptyline, carbamazepine, cyclosporine, clozapine, digoxin, phenobarbital, phenytoin, gentamycin, midazolam, acetaminophen, theophylline, tobramycin, valproic acid and vancomycin. The sieving coefficient (SC) was calculated by $\mathrm{SC}=$ ( $2 \mathrm{x}$ concentration ultrafiltrate) / (concentration post membrane + concentration pre membrane).

Results: All four parameters; \% PB, $\log \mathrm{P}, \mathrm{C}$ and $\mathrm{Mw}$ contributed statistically significant $(\mathrm{p}<0,05)$ to the $\mathrm{SC}$. The regression line, explaining $75 \%$ of the variance $\left(\mathrm{R}^{2}=0,751\right)$ is:

$\mathrm{SC}=0,826-0,06 \% \mathrm{~PB}-0,055 \log \mathrm{P}-0,085 \mathrm{C}+0,000095 \mathrm{MW}$ 
Conclusion: Based on the ex-vivo experiments a mathematical model has been extracted to predict a-priori the sieving coefficient of drugs during CVVH with an AN69 high-flux membrane on the base of protein binding, charge, $\log \mathrm{P}$ and molecular weight. This model has to be validated in vivo and can then be used, if valid, for drugs which physico-chemical properties in the ranges studied: p\%PB $0-98 \%, \log \mathrm{P}-7,32-7$, Mw $151-1500, \mathrm{C}-1-3$.
Mueller B, et al. Artificial organs 2003; 27(9): 808-14. Reetze-Bonorden P et al. Clin Pharmacokinet 1993; 24(5): 362-79.

\section{Risk calculation for hyperkalemia in heart failure patients}

\author{
T. L. J. Vereijken,' L. Bellersen, ${ }^{2}$ J. M. M. Groenewoud ${ }^{3}$ \& \\ C. Kramers ${ }^{1}$ \\ ${ }^{7}$ Depts. Pharmacology-Toxicology, ${ }^{2}$ Cardiology and ${ }^{3}$ Medical \\ Technology Assesment, Radboud University Hospital, Nijmegen, \\ The Netherlands
}

Introduction: Since it was propagated to use aldosterone inhibition (Aldo i) on top of ACE inhibitors (ACEi) for the treatment of heart failure, the incidence of hospitalization and death because of hyperkalemia has risen. We aimed to develop a model to estimate the risk of hyperkalemia in patients treated for heart failure in a tertiary reference hospital and to identify precipitating factors.

Methods: 125 CHF patients were studied retrospectively between January 2002 and April 2006. Thirty of these patients developed episodes of hyperkalemia ( $\mathrm{K} \geq 5.5 \mathrm{mmol} / \mathrm{l})$. Both groups were compared for possible risk factors for hyperkalemia (age, GFR, NYHA class, diabetes mellitus, ejection fraction and medication use (ACEi, angiotensin receptor blockers, Aldo i)). Moreover it was assessed whether a precipitating factor for the hyperkalemic episode could be identified.
Results: On multivariate logistic regression analysis DM (OR 2.9; $95 \% \mathrm{CI}=1.05-8.3 \mathrm{p}=0.041), \mathrm{GFR}<45 \mathrm{ml} / \mathrm{min}(\mathrm{OR} 4.1 ; 95 \% \mathrm{CI}$ $=1.6-10.5 \mathrm{p}=0.004)$ and NYHA class III-IV (OR 2.4; 95\% CI = $0.9-6.3 \mathrm{p}=0.086$ ) were independently associated with hyperkalemia, whereas age, ejection fraction and medication sort and dose, were not. Based on these ORs a model was designed which could predict hyperkalemia with a sensitivity of $90 \%$ and a specificity of $47 \% .38 \%$ of episodes of hyperkalemia were precipitated by periods of dehydration (diarrhoea, fever) or change of medication, whereas in $62 \%$ no precipitating factor could be identified. In all but one hyperkalemic episode there was concomitant transient loss of renal function (i.e rise of creatinin of at least 25\%).

Conclusion: We identified kidney function, diabetes mellitus and heart failure class as independent risk factors of hyperkalemia. The model based on these risk factors gives only a modest prediction of hyperkalemia. Moreover, the majority of the hyperkalemic episodes develop without precipitating factor, although the concomitant transient loss of renal function suggest there is unnoticed transient dehydration in these subjects. These findings imply that heart failure patients in a third line reference hospital should be very closely monitored to minimize the risk for hyperkalemia.

\section{Development strategy and potential impact on medication safety for clinical rules: the lithium case}

S. J. W. Wessels-Basten, ${ }^{1}$ A. M. J. W. Hoeks, ${ }^{1}$ R. J. E. Grouls, ${ }^{1}$

P. J. Helmons, ${ }^{1}$ E. W. Ackerman' ${ }^{1}$ \& H. H. M. Korsten ${ }^{2}$

${ }^{\prime}$ Dept. of Clinical Pharmacy, ${ }^{2}$ Anesthesiology, Catharina Hospital

Eindhoven, PO Box 1350, 5602 ZA Eindhoven, The

Netherlands

Introduction: Prescribing, administration and monitoring of drugs are complex and error sensitive processes. Physicians have to take into account many drug- and patient specific characteristics continuously during therapy. Appropriate use of information technology, especially the introduction of clinical decision support systems (CDSS) can substantially reduce medication error rates and improve patient safety $[1,2]$. Strategies for the development of clinical rules used in these CDSS systems are not available.

Purpose: The aim of this study was: (1) to evaluate a strategy for the development of clinical rules and (2) to determine the potential impact on medication safety of these clinical rules. Lithium therapy was chosen as a test concept.

Methods: For the development of clinical rules we followed a Plan - Do - Check - Act (PDCA) cycle with a panel of experts. Standard literature sources were screened for patient characteristics, monitoring parameters and other characteristics that have to be taken into account during lithium therapy. We translated this information into clinical rules for the CDSS system Gaston ${ }^{\circledR \#}$ (plan). These rules were retrospectively tested on patients admitted to the Catharina-hospital Eindhoven in 2004 and 2005 using lithium (do). Signals were checked in the electronic patients record on: (1) appropriateness and (2) the action undertaken by the physician compared to the action warranted by the CDSS. For every rule the Positive Predictive Value (PPV) was determined: number of appropriate signals produced /total number of signals. When the action undertaken was not right according to the clinical rules we scored this as a Potential Adverse Drug Event (PADE) (check). Results were discussed with an expert panel and the clinical rules adjusted according their consensus (act).

Results: The PDCA strategy with an expert panel resulted in a package of clinical rules for patients starting with, or using lithium therapy. To design the definite clinical rules the input of the expert team was essential, because literature left to much room for interpretation in clinical practice. The clinical rules were tested on 38 patients starting with and 93 patients using lithium in 2004 or 2005. The PPV's of these clinical rules varied from 0.2 to 1.0 (mean 0.8 ). We identified 81 PADE's in 71 patients, that could have been prevented when the CDSS would have been active. Most important PADE's were: 1) omitted determination of lithium plasma levels after reduction of creatinin clearance $<50 \mathrm{ml} / \mathrm{min}$ (4 times) and starting or stopping of an interacting drug (17 times); 2) determination of lithium, Thyroid Stimulating Hormone (TSH), leucocyte and 
calcium plasma levels every 3 or 6 months (resp. 17, 6, 2 and 4 times). An important limitation for the creation of higher PPV's and for the identification of PADE's was the lack of outpatient data. Further research is needed to show that this system is clinically applicable.

Conclusion: A strategy of PDCA combined with an expert panel is a prerequisite for the development of clinical rules for drugs in a CDSS. We demonstrated that a CDSS is able to identify patients with potential adverse drug events. Therefore we conclude that application of CDSS can have a relevant impact on medication safety in clinical practice.

1 Bates et al. JAMA 2001; 8: 299-308.

2 Kaushal et al. Arch Intern Med 2003; 163: 1409-1416.

${ }^{\#}$ Gaston $^{\circledR}$ : P.A.de Clerq, MEDECS b.v. www.medecs.nl

\begin{abstract}
Non-detectable levels of 6-thioguanine nucleotides and 6-methylmercaptopurine in a patient treated with azathioprine: a case report

D. R. Wong, ${ }^{1}$ M. O. den Dulk, ${ }^{2}$ L. J. J. Derijks, ${ }^{3}$ E. H. K. M.

Gemmeke $^{4}$ \& P. M. Hooymans'

'Department of Clinical Pharmacy, Maasland Hospital, Sittard;

${ }^{2}$ Department of Internal Medicine, St. Jans Gasthuis Hospital,

Weert; ${ }^{3}$ Department of Clinical Pharmacy, Maxima Medical

Centre, Veldhoven; ${ }^{4}$ Department of Clinical Pharmacy, St. Jans

Gasthuis Hospital, Weert; The Netherlands
\end{abstract}

Introduction: Azathioprine (AZA) is a thiopurine prodrug clinically used for immunosuppression in the treatment of auto-immune inflammatory diseases and in regimens of organ transplantations. The pharmacological action of AZA is based on the formation of 6mercaptopurine (6-MP), which is metabolised into a variety of active thiopurine-nucleotide metabolites. We report the case of a 55 year old woman (bodyweight $30 \mathrm{~kg}$ ) with chronic pancreatitis, weight loss, and progressive elevation of liver transaminases and serum amylase.

Case description: The woman was treated with prednisolone $(30 \mathrm{mg}$ $1 \mathrm{dd}$; tapered $5 \mathrm{mg}$ each week) and AZA ( $75 \mathrm{mg} 1 \mathrm{dd} ; 5$ weeks later $150 \mathrm{mg} 1 \mathrm{dd}$ ). Despite good patient-compliance verified during hospital-stay, none of the active metabolites of AZA, 6-thioguaninenucleotides (6TGN) and 6-methylmercaptopurine ribonucleotides (6MMPR), were detected in erythrocytes. After two months of treatment clinical improvement was achieved, but no normalisation of laboratory parameters. Subsequently, AZA was switched to 6-MP 75 mg $1 \mathrm{dd}$, and allopurinol $100 \mathrm{mg} 1 \mathrm{dd}$ was added. After one week the 6 TGN level was $616 \mathrm{pmol} / 8 \times 10^{8}$ red blood cells $(\mathrm{RBC})$, the $6 \mathrm{MMPR}$ level was $1319 \mathrm{pmol} / 8 \times 10^{8} \mathrm{RBC}$.

Two weeks later the 6 TGN level was $1163 \mathrm{pmol} / 8 \times 10^{8} \mathrm{RBC}$ and the 6MMPR level $10015 \mathrm{pmol} / 8 \times 10^{8} \mathrm{RBC}$.

These 6TGN and 6MMPR levels were higher than the upper limits of the therapeutic ranges $\left(500 \mathrm{pmol} / 8 \times 10^{8} \mathrm{RBC}\right.$ and $5700 \mathrm{pmol} / 8$ $\times 10^{8} \mathrm{RBC}$, respectively). Therefore, treatment with 6-MP was discontinued. A week later the 6TGN and 6MMPR levels decreased to $686 \mathrm{pmol} / 8 \times 10^{8} \mathrm{RBC}$ and $4027 \mathrm{pmol} / 8 \times 10^{8} \mathrm{RBC}$, respectively.

Genotyping of the enzym thiopurine S-methyl transferase (TPMT) revealed a wild-type TPMT $(* 1 / * 1)$ genotype.

Discussion: To our knowledge this is the first report of a patient who was not able to form detectable active thiopurine metabolites on the treatment with AZA.

AZA is normally rapidly and almost completely converted to 6MP and methylnitroimidazole in the liver. 6-MP is then further catabolised by xanthine-oxidase (XO) and TPMT or anabolised by hypoxanthine phosphoribosyl transferase (HPRT). Remarkably, treatment with 6-MP in combination with the XO-inhibitor allopurinol resulted in a myelotoxic 6TGN level in the first week after start of treatment.

There are two hypotheses on the mechanism by which this finding could be explained. First, an 'ultra-high' $\mathrm{XO}$ activity results in a direct and complete conversion of 6-MP into 6-thiouric acid. Secondly, AZA is not converted into 6-MP. Consequently, there is no formation of active thiopurine nucleotides out of 6-MP.

Also, a combination of these two mechanisms could lead to a decreased formation of active thiopurine metabolites.

Conclusion: Our finding indicates non-conventional insights in the biotransformation of AZA contributing to an interindividual variation in thiopurine metabolism. 